Article

\title{
Improving the Fermentation Production of the Individual Key Triterpene Ganoderic Acid Me by the Medicinal Fungus Ganoderma lucidum in Submerged Culture
}

\section{Gao-Qiang Liu ${ }^{1,2, *}$, Xiao-Ling Wang ${ }^{1}$, Wen-Jun Han ${ }^{1}$ and Qin-Lu Lin ${ }^{2}$}

1 Hunan Provincial Key Laboratory of Forestry Biotechnology, College of Life Science and Technology, Central South University of Forestry and Technology, Changsha 410004, China; E-Mails:wxl_edu@yahoo.com.cn (X.-L.W.); qinxinhuzhu@tom.com (W.-J.H.)

2 National Engineering Laboratory for Rice and By-product Further Processing, Central South University of Forestry and Technology, Changsha 410004, China; E-Mail: phdqlin@yahoo.cn

* Author to whom correspondence should be addressed; E-Mail: gaoliuedu@yahoo.com.cn; Tel./Fax: +86-731-8562-3498.

Received: 8 October 2012; in revised form: 17 October 2012 / Accepted: 18 October 2012 / Published: 24 October 2012

\begin{abstract}
Enhanced ganoderic acid Me (GA-Me, an important anti-tumor triterpene) yield was attained with the medicinal fungus Ganoderma lucidum using response surface methodology (RSM). Interactions were studied with three variables, viz. glucose, peptone and culture time using a Central Composite Design (CCD). The CCD contains a total of 20 experiments with the first 14 experiments organized in a fractional factorial design, with the experimental trails from 15 to 20 involving the replications of the central points. A polynomial model, describing the relationships between the yield of GA-Me and the three factors in a second-order equation, was developed. The model predicted the maximum GA-Me yield of $11.9 \mathrm{mg} \cdot \mathrm{L}^{-1}$ for glucose, peptone, culture time values of $44.4 \mathrm{~g} \cdot \mathrm{L}^{-1}$, $5.0 \mathrm{~g} \cdot \mathrm{L}^{-1}, 437.1 \mathrm{~h}$, respectively, and a maximum GA-Me yield of $12.4 \mathrm{mg} \cdot \mathrm{L}^{-1}$ was obtained in the validation experiment, which represented a $129.6 \%$ increase in titre compared to that of the non-optimized conditions. In addition, $11.4 \mathrm{mg} \cdot \mathrm{L}^{-1}$ of GA-Me was obtained in a 30-L agitated fermenter under the optimized conditions, suggesting the submerged culture conditions optimized in the present study were also suitable for GA-Me production on a large scale.
\end{abstract}


Keywords: medicinal fungi; Ganoderma lucidum; submerged culture; response surface methodology; ganoderic acid Me

\section{Introduction}

Ganoderma lucidum (Leyss. ex Fr.) Karst ("Ling-zhi" in Chinese and "Reishi" in Japanese) is a famous medicinal mushroom that has been used as a traditional medicine for a long time in Asia [1]. The potential medicinal value and wide acceptability of this edible mushroom have attracted intense interest in the search for its biologically active substances during the last 30 years [2-4]. Nowadays, G. lucidum and related products are widely used not only as health foods, but also clinical drugs for the prevention and treatment of hepatopathy, chronic hepatitis, nephritis, gastric ulcer, hypertension, arthritis, neurasthenia, insomnia, asthma, acute and chronic bronchitis, leucopoenia, and cancer $[5,6]$.

Modern chemistry studies show that G. lucidum contains a variety of phytochemicals. Some of the potent biologically active compounds that have been shown to possess diverse and potentially significant pharmacological activities are the bitter triterpenes (especially ganoderic acids, GAs) [7-10]. Since the first discovery of ganoderic acids A and B (GA-A, GA-B), over 150 types of triterpenes have been isolated from various parts of G. lucidum [11], among which GA-Me has received considerable attention due to its conspicuous pharmacological properties, especially anticancer activity. GA-Me effectively inhibited tumor growth, lung metastasis [12], and tumor invasion through down-regulating matrix metalloproteinase 2/9 (MMP2/9) gene expression [13]. It was found that GA-Me depressed the viability of tumor cells at much lower concentrations than against normal cells [14], making it an effective potential therapeutic drug for the prevention and treatment of tumors in the clinic. In addition, GA-Me was demonstrated to inhibit cholesterol synthesis $[10,15]$.

Currently, submerged fermentation of G. lucidum is viewed as a promising alternative for efficient production of GAs because it usually takes several months to cultivate the fruiting body of G. lucidum and it is also difficult to control the product quality during its cultivation [16,17]. Many researchers have focused on studying fermentation conditions to accelerate mycelial growth and optimize the production of GA by mycelia fermentation [16-19]. However, most of those previous reports were about total crude GAs production based on UV absorbance measurements [16-19], and the total GAs are a complicated mixture which usually also contains other organic acids [20] so the content may not accurately reflect GA accumulation. In addition, not all the GAs are bioactive. Therefore, investigation of the individual key GA production in submerged culture is necessary. In our previous study, we established the key factors (glucose, peptone and culture time) for total crude GA production in submerged culture [17]. The aim of this work was to improve the fermentation production of individual GA-Me based on the three key factors and study the effects of the mutual interactions of these factors on individual GA-Me production using a central composite design (CCD) and response surface methodology (RSM). 


\section{Results and Discussion}

\subsection{Production of GA-Me under Non-Optimized Culture Conditions}

According to the culture media and fermentation conditions used for the maximum production of total crude GAs in our previous work [17] (as described in the Experimental section: basic fermentation conditions), triplicate experiments were carried out to examine the individual GA-Me production yield. The mycelial dry weight (DW) reached $13.1 \mathrm{~g} \cdot \mathrm{L}^{-1}$, and a $5.4 \mathrm{mg} \cdot \mathrm{L}^{-1}$ of GA-Me production yield was obtained in $G$. lucidum cultures, of which, $2.8 \mathrm{mg} \cdot \mathrm{L}^{-1}$ was obtained in the mycelia and $2.6 \mathrm{mg} \cdot \mathrm{L}^{-1}$ was collected from the fermented supernatant (after removal of mycelia by centrifugation). The content of GA-Me of mycelia was $21.3 \mu \mathrm{g} \cdot 100 \mathrm{mg}^{-1} \mathrm{DW}$.

\subsection{Optimization of GA-Me Production by CCD and RSM}

In our earlier study, glucose, peptone and culture time were the key factors for GA production [17]. In the present work, the levels of the three key factors were further optimized by CCD and RSM to improve the production yield of individual GA-Me.

\subsubsection{Response Surface Analysis for the Optimization of Culture Condition Levels}

A central composite design (CCD) was used in the optimization of GA-Me production. The ranges and the levels of the variables investigated in this study are given in Table 1.

Table 1. Experimental range and levels of the independent variables of ganoderic acid Me (GA-Me) optimization.

\begin{tabular}{cccccc}
\hline Independent & \multicolumn{5}{c}{ Range and levels } \\
\cline { 2 - 6 } variables & $-\mathbf{1 . 6 8 2}$ & $\mathbf{- 1}$ & $\mathbf{0}$ & $\mathbf{1}$ & $\mathbf{1 . 6 8 2}$ \\
\hline Glucose, $x_{1}\left(\mathrm{~g} \cdot \mathrm{L}^{-1}\right)$ & 36.59 & 40.0 & 45.0 & 50.0 & 53.41 \\
Peptone, $x_{2}\left(\mathrm{~g} \cdot \mathrm{L}^{-1}\right)$ & 3.32 & 4.00 & 5.0 & 6.00 & 6.68 \\
Fermentation time, $x_{3}(\mathrm{~h})$ & 265.91 & 300 & 13 & 400 & 434.09 \\
\hline
\end{tabular}

The results of CCD experiments for GA-Me production are presented in Table 2. Table 3 shows the analysis of variance for the experiment. The Fisher's F-test with a very low probability value $\left[\left(P_{\text {model }}>F\right)=0.0002\right]$ for the total model indicated the model was highly significant. The goodness of fit of the model was examined by the coefficient of determination $\left(R^{2}=0.9257\right)$, which implied that more than $92 \%$ of the sample variation was attributable to the variables and only $7.43 \%$ of the total variance could not be explained by the model. The adjusted determination coefficient (Adj. $R^{2}=0.8570$ ) was also satisfactory to confirm the significance of the model.

Table 4 reveals the analysis of variance (ANOVA) for three key factors on the production of GA-Me. The smaller the $P$-value, the more significant is the corresponding factor. $X_{3}$ (culture time) had extremely significant effect on GA-Me production $(P<0.01)$, followed by $x_{2}$ (peptone concentration), suggesting culture time and peptone concentration are very important for GA-Me production in submerged fermentation of G. lucidum. 
Table 2. The central composite design matrix and the response of ganoderic acid Me (GA-Me) of G. lucidum.

\begin{tabular}{|c|c|c|c|c|}
\hline \multirow{2}{*}{ Runs } & \multicolumn{3}{|c|}{ Coded values } & \multirow{2}{*}{$\begin{array}{c}Y_{G A-M e} \\
\left(\mathrm{mg} \cdot \mathrm{L}^{-1}\right)\end{array}$} \\
\hline & Factor $x_{1}$ & Factor $x_{2}$ & Factor $x_{3}$ & \\
\hline 1 & -1 & -1 & -1 & 4.9 \\
\hline 2 & -1 & -1 & 1 & 9.9 \\
\hline 3 & -1 & 1 & -1 & 4.1 \\
\hline 4 & -1 & 1 & 1 & 7.4 \\
\hline 5 & 1 & -1 & -1 & 6.9 \\
\hline 6 & 1 & -1 & 1 & 10.5 \\
\hline 7 & 1 & 1 & -1 & 5.1 \\
\hline 8 & 1 & 1 & 1 & 6.7 \\
\hline 9 & -1.682 & 0 & 0 & 6.1 \\
\hline 10 & 1.682 & 0 & 0 & 8.1 \\
\hline 11 & 0 & -1.682 & 0 & 7.0 \\
\hline 12 & 0 & 1.682 & 0 & 6.7 \\
\hline 13 & 0 & 0 & -1.682 & 3.1 \\
\hline 14 & 0 & 0 & 1.682 & 11.2 \\
\hline 15 & 0 & 0 & 0 & 8.9 \\
\hline 16 & 0 & 0 & 0 & 8.8 \\
\hline 17 & 0 & 0 & 0 & 8.8 \\
\hline 18 & 0 & 0 & 0 & 8.5 \\
\hline 19 & 0 & 0 & 0 & 7.9 \\
\hline 20 & 0 & 0 & 0 & 8.2 \\
\hline
\end{tabular}

Table 3. Analysis of variance (ANOVA) for the full quadratic model for optimization of ganoderic acid Me (GA-Me) production in G. lucidum.

\begin{tabular}{cccccc}
\hline Regression & DF & Sum of Squares & $\boldsymbol{R}$-Square & $\boldsymbol{F}$ value & $\boldsymbol{P r}>\boldsymbol{F}$ \\
\hline Linear & 3 & 63.2143 & 0.7614 & 34.16 & $<0.0001^{* *}$ \\
Quadratic & 3 & 10.0708 & 0.1213 & 5.44 & $0.0177^{*}$ \\
Crossproduct & 3 & 3.5738 & 0.0430 & 1.93 & 0.1886 \\
Total model & 9 & 76.8589 & 0.9257 & 13.84 & $0.0002 * *$ \\
\hline
\end{tabular}

$R^{2}=0.9257$; Adj. $R^{2}=0.8570 ; * *$ Significant at 0.01 level, $*$ Significant at 0.05 level.

Table 4. Analysis of variance (ANOVA) for three factors for optimization of ganoderic acid Me (GA-Me) production of G. lucidum.

\begin{tabular}{cccccc}
\hline Factor & DF & Sum of Squares & Mean Square & $\boldsymbol{F}$ value & $\boldsymbol{P r}>\mathbf{F}$ \\
\hline x1 & 4 & 8.3684 & 2.0921 & 3.39 & 0.0534 \\
x & 4 & 13.8737 & 3.4684 & 5.62 & $0.0123 *$ \\
x 3 & 4 & 60.1597 & 15.0399 & 24.38 & $<0.0001 * *$ \\
\hline
\end{tabular}

** Significant at 0.01 level, * Significant at 0.05 level.

The significance of each coefficient was determined by Student's $t$-test and $P$-value, which is listed in Table 5 The larger the magnitude of $t$-test and smaller the $P$-value, the more significant is the corresponding coefficient. The polynomial model for GA-Me yield $Y_{\mathrm{GA}-\mathrm{Me}}$ was regressed by all the 
terms and was expressed by Equation (1). The regression equation showed that the GA-Me yield was an empirical function of test variables in coded unit.

$$
\begin{gathered}
Y_{\mathrm{GA}-\mathrm{Me}}=8.5169+0.4586 x_{1}-0.6886 x_{2}+1.9860 x_{3}-0.2875 x_{1} x_{2}-0.3875 x_{1} x_{3}- \\
0.4625 x_{2} x_{3}-0.5021 x_{1}{ }^{2}-0.5905 x_{2}{ }^{2}-0.4844 x_{3}{ }^{2}
\end{gathered}
$$

where $Y_{\mathrm{GA}-\mathrm{Me}}$ is the predicted GA-Me yield, $x_{1}$ glucose, $x_{2}$ peptone, and $x_{3}$ is culture time.

Table 5. Results of regression analysis of a predictive polynomial model for optimization of ganoderic acid Me (GA-Me) of G. lucidum.

\begin{tabular}{cccccc}
\hline Parameter & DF & Coefficients estimated & Standard error & $\boldsymbol{t}$ value & $\boldsymbol{P r}>|\boldsymbol{t}|$ \\
\hline Intercept & 1 & 8.5169 & 0.3203 & 26.59 & $<0.0001^{* *}$ \\
$x_{1}$ & 1 & 0.4586 & 0.2125 & 2.16 & 0.0563 \\
$x_{2}$ & 1 & -0.6886 & 0.2125 & -3.24 & $0.0089 * *$ \\
$x_{3}$ & 1 & 1.9860 & 0.2125 & 9.34 & $<0.0001 * *$ \\
$x_{1} x_{1}$ & 1 & -0.5021 & 0.2069 & -2.43 & $0.0356 *$ \\
$x_{1} x_{2}$ & 1 & -0.2875 & 0.2777 & -1.04 & 0.3249 \\
$x_{2} x_{2}$ & 1 & -0.5905 & 0.2069 & -2.85 & $0.0171 *$ \\
$x_{1} x_{3}$ & 1 & -0.3875 & 0.2777 & -1.40 & 0.1931 \\
$x_{2} x_{3}$ & 1 & -0.4625 & 0.2777 & -1.67 & 0.1268 \\
$x_{3} x_{3}$ & 1 & -0.4844 & 0.2069 & -2.34 & $0.0412 *$ \\
\hline
\end{tabular}

** Significant at 0.01 level, * Significant at 0.05 level.

Equation (1) also reveals that culture time $\left(x_{3}\right)$ had a strong positive linear effect on the response $(P<0.01)$ of $Y_{\mathrm{GA}-\mathrm{Me}}$ as it had the largest coefficient, followed by peptone $\left(x_{2}\right)$, which showed a significant negative linear effect $(P<0.01)$. However, glucose $\left(x_{1}\right)$ had no significant effect on GA-Me production at the tested concentrations $(P>0.05)$, and the above three variables also indicated negative quadric effects on GA-Me yield $(P<0.05)$. No significant interactions were noted between any two of the three variables $(P>0.05)$.

\subsubsection{Interaction among the Factors and Selection of Their Optimum Levels}

The 3D response surface is generally the graphical representation of the regression equation. Figures 1-3 represent the 3D response surfaces for the optimization of culture condtion of GA-Me production. Each figure presented the effect of two variables on the production of GA-Me, while another variable was held at zero level (coded value).

From the response surface plots, it is easy to understand the interactions between two nutrients and also to locate their optimum levels. It can be seen from Figures 1 and 2 that the yield of GA-Me was high when the concentrations of peptone were in the range of 3.6-4.8 g. $\mathrm{L}^{-1}$ and glucose in the range of 45-51 g. $\mathrm{L}^{-1}$ synchronously. Figures 2 and 3 showed that GA-Me yield increased upon increasing the culture time from 270 to $420 \mathrm{~h}$. The model predicted a maximum GA-Me yield of $11.9 \mathrm{mg} \cdot \mathrm{L}^{-1}$ for glucose, peptone, culture time values of $44.4 \mathrm{~g} \cdot \mathrm{L}^{-1}, 5.0 \mathrm{~g} \cdot \mathrm{L}^{-1}, 437.1 \mathrm{~h}$, respectively. 
Figure 1. The surface plot of the combined effects of glucose and peptone on $\mathrm{Y}_{1}(\mathrm{GA}-\mathrm{Me})$ production. Fixed level: culture time $=0$ (coded value).

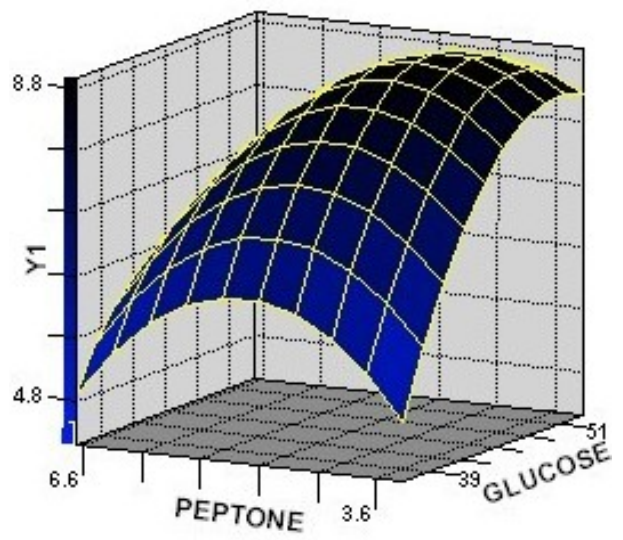

Figure 2. The surface plot of the combined effects of glucose and culture time on $\mathrm{Y}_{1}$ (GA-Me) production by G. lucidum. Fixed level: peptone concentration $=0$ (coded value).

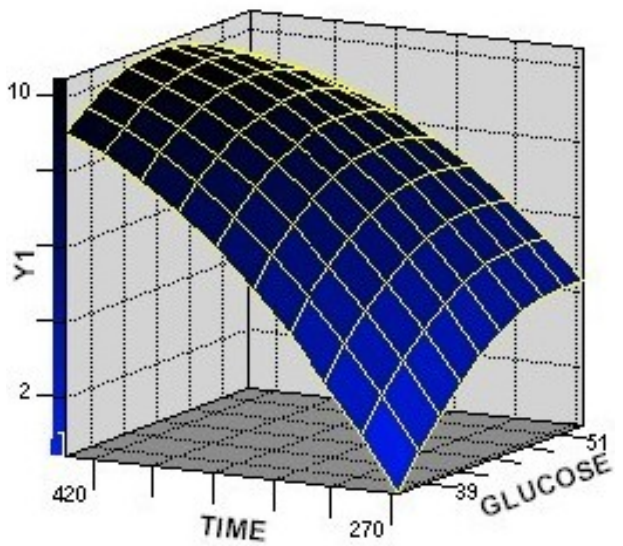

Figure 3. The surface plot of the combined effects of peptone and culture time on $\mathrm{Y}_{1}$ (GA-Me) production by G. lucidum. Fixed level: glucose concentration = 0 (coded value).

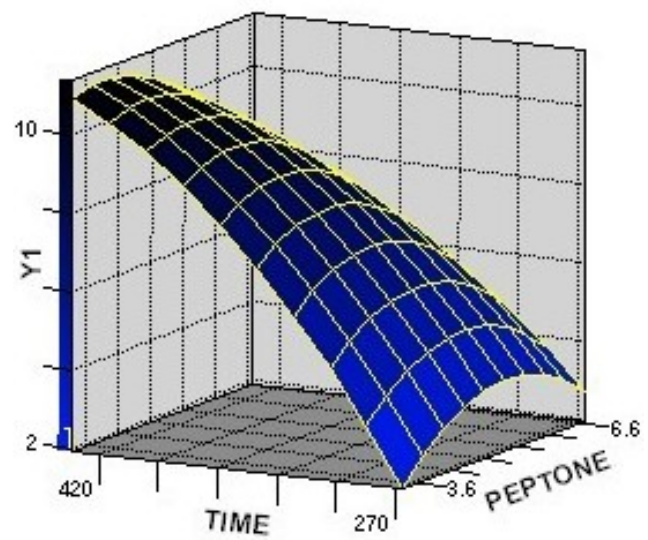




\subsubsection{Verification of the Models in Flask Culture and Bioreactor Culture}

Triplicate experiments were carried out to verify the availability and accuracy of the model (Equation (1)) for GA-Me production in flask shake culture. The results are shown in Table 6. Under the calculated optimum culture conditions, a cell density of $11.3 \mathrm{~g} \cdot \mathrm{L}^{-1}$ (by dry weight, DW) was obtained, and the content of GA-Me in mycelial cell was $42.7 \mu \mathrm{g} \cdot 100 \mathrm{mg}^{-1} \mathrm{DW}$ (also for $4.8 \mathrm{mg} \cdot \mathrm{L}^{-1}$ based on $11.3 \mathrm{~g} \cdot \mathrm{L}^{-1}$ of mycelia), and that in fermented supernatant (removal of mycelia by centrifugation) it was $7.6 \mathrm{mg} \cdot \mathrm{L}^{-1}$, that is, a $12.4 \mathrm{mg} \cdot \mathrm{L}^{-1}$ of GA-Me production yield was obtained in G. lucidum cultures, which represented a $129.6 \%$ increase in titre compared to the the non-optimized culture conditions $\left(5.4 \mathrm{mg} \cdot \mathrm{L}^{-1}\right)$, and was also in agreement with the predicted value $\left(11.9 \mathrm{mg} \cdot \mathrm{L}^{-1}\right)$. The good agreement between the predicted and experimental results verifies the validity of the model and the existence of an optimum point. The feasibility of the regression model was further tested in a 30-L scaled fermenter under the optimized culture condition. A maximum yield $\left(11.4 \mathrm{mg} \cdot \mathrm{L}^{-1}\right)$ of GA-Me was obtained (Table 6), suggesting the culture condition optimized in the present study was also suitable for GA-Me production in a large scale.

Table 6. Verification results for ganoderic acid Me (GA-Me) production under the optimized culture condition by G. lucidum.

\begin{tabular}{cccccc}
\hline Culture systems & $\begin{array}{c}\text { Cell } \\
\text { density } \\
\left(\mathbf{g} \cdot \mathbf{L}^{-\mathbf{1}}\right)\end{array}$ & $\begin{array}{c}\text { GA-Me } \\
\text { content in Cell } \\
(\boldsymbol{\mu} \mathbf{g} / \mathbf{1 0 0} \mathbf{~} \mathbf{g ~ D W})\end{array}$ & $\begin{array}{c}\text { GA-Me } \\
\text { production } \\
\text { in Cell } \\
\left(\mathbf{m g} \cdot \mathbf{L}^{-\mathbf{1}}\right)\end{array}$ & $\begin{array}{c}\text { GA-Me } \\
\text { production } \\
\text { in fermented } \\
\text { broth }\left(\mathbf{m g} \cdot \mathbf{L}^{-\mathbf{1}}\right)\end{array}$ & $\begin{array}{c}\text { Final glucose } \\
\text { concentration } \\
\left(\mathbf{g} \cdot \mathbf{L}^{-\mathbf{1}} \mathbf{)}\right.\end{array}$ \\
\hline $\begin{array}{c}\text { Flask culture } \\
\text { Bioreactor culture }\end{array}$ & $11.3 \pm 0.2$ & $42.7 \pm 0.4$ & $4.8 \pm 0.7$ & $7.6 \pm 0.2$ & $4.7 \pm 0.3$ \\
\hline
\end{tabular}

Until now, GAs were mainly extracted from the solid cultivated fruiting bodies of G. lucidum [21]. It usually takes several months to cultivate the fruiting body, and the yield of GAs is very low [11]. Consequently, cell culture of G. lucidum is recognized as a promising alternative for efficient production of GAs [17-19]. Although it has been reported that GA accumulation was influenced by the culture medium and process conditions of G. lucidum, many studies were about total GAs production [16-19], and there are few reports available for individual GAs. Xu et al. studied the production of individual ganoderic acids (GA-Me, GA-T, GA-S and GA-Mk) by G. lucidum cell culture [22]. It was found that GA-Me yield in liquid static culture was higher than that obtained in liquid shaking culture. The GA-Me content in static culture was in the range of 9.6-11.5 mg. $\mathrm{L}^{-1}$ (cell density: 10-12.5 $\mathrm{g} \cdot \mathrm{L}^{-1}$; GA-Me content: $96 \mu \mathrm{g} \cdot 100 \mathrm{mg}^{-1} \mathrm{DW}$ ), and the GA-Me content was higher than that obtained in the present study. However, the GA-Me yield was very low in shaking flask culture with a lactose-based culture medium, and the content of GA-Me in the mycelium was about $10.1 \mu \mathrm{g} \cdot 100 \mathrm{mg}^{-1} \mathrm{DW}$, which is lower than that in the present work. Nevertheless the content of GA-Me in fermented supernatant (extracellular GA-Me) was not determined by Xu et al. In the present study, we obtained $7.6 \mathrm{mg} \cdot \mathrm{L}^{-1}$ of GA-Me from fermented supernatant, and $4.8 \mathrm{mg} \cdot \mathrm{L}^{-1}$ of GA-Me from mycelia, suggesting some quantity of GA-Me was excreted into the fermented broth during the culture process, a phenomenon also observed in several previous studies [17,21,23,24]. In addition, the optimized culture condition was successfully applied in GA-Me production in an agitated 30-L 
fermenter. Generally, in submerged mushroom cultures, it is necessary to agitate the culture broth in order to obtain good mixing and thereby promote heat and mass transfer [25]. However, agitation also creates shear force. The shear stress exerted by the impeller blades in an agitated fermenter usually reduces and modifies the mycelium pellet growth in terms of diameter, circularity and compactness, and the accumulation of metabolites. In this work, the mycelium showed no significant damage caused by the shear forces with a speed of $150 \mathrm{rpm} \cdot \mathrm{min}^{-1}$, and $9.1 \mathrm{~g} \cdot \mathrm{L}^{-1}$ of mycelium pellets and a $11.4 \mathrm{mg} \cdot \mathrm{L}^{-1}$ titre of GA-Me was obtained, suggesting GA-Me could be also produced under the optimized culture conditions in a large-scale agitated bioreactor.

\section{Experimental}

\subsection{Microorganism}

Stock cultures of G. ludicum SCIM 0006 were obtained from the Strain Collection of Industrial Microorganisms (SCIM), Central South University of Forestry \& Technology (Changsha, China), and maintained on potato-dextrose-agar slants, stored at $4{ }^{\circ} \mathrm{C}$.

\subsection{Liquid Shake Culture}

G. lucidum SCIM 0006 was grown in a $250-\mathrm{mL}$ flask containing $80 \mathrm{~mL}$ seed culture medium (see below) at $30{ }^{\circ} \mathrm{C}$ for 8 days with shaking at $160 \mathrm{rpm}$. This was then inoculated at $12 \%(\mathrm{v} / \mathrm{v})$ into the fermentation medium incubated at $28^{\circ} \mathrm{C}$ for different days for optimization of GA-Me production. The seed culture medium composed of (in $\mathrm{g} \cdot \mathrm{L}^{-1}$ ): glucose (40.0), peptone (4.0), $\mathrm{KH}_{2} \mathrm{PO}_{4}(0.75)$, $\mathrm{MgSO}_{4} \cdot 7 \mathrm{H}_{2} \mathrm{O}(0.45)$ and vitamin $\mathrm{B}_{1}(0.01)$. The fermentation medium composed of $\left(\mathrm{g} \cdot \mathrm{L}^{-1}\right)$ : glucose (36.595-3.41), peptone (3.326-68), $\mathrm{KH}_{2} \mathrm{PO}_{4}(0.75), \mathrm{MgSO}_{4} \cdot 7 \mathrm{H}_{2} \mathrm{O}(0.50)$ and vitamin $\mathrm{B}_{1}(0.01)$. The basic fermentation conditions were as follows: glucose $\left(38 \mathrm{~g} \cdot \mathrm{L}^{-1}\right)$, peptone $\left(4.5 \mathrm{~g} \cdot \mathrm{L}^{-1}\right), \mathrm{KH}_{2} \mathrm{PO}_{4}(0.75)$, $\mathrm{MgSO}_{4} \cdot 7 \mathrm{H}_{2} \mathrm{O}(0.45)$ and vitamin $\mathrm{B}_{1}(0.01)$; culture time $300 \mathrm{~h}$; culture temperature $28{ }^{\circ} \mathrm{C}[17]$.

\subsection{Bioreactor Culture}

The bioreactor culture was carried out by a similar method described in our previous work [26] in an agitated 30-L fermenter (New Brunswick Scientific Co., Enfield, CT, USA), under the following conditions: medium volume $20 \mathrm{~L}$, inoculation volume $12 \%(\mathrm{v} / \mathrm{v})$, temperature $28{ }^{\circ} \mathrm{C}$, aeration rate $6.0 \mathrm{vvm}$, and agitation speed $150 \mathrm{rpm}$.

\subsection{Determination of Dry Cell Weight}

Samples collected from flasks were filtered using a 40-mesh stainless sieve and the mycelium was harvested. Mycelial biomass was collected by centrifuging the mycelium at 8,000 rpm for $15 \mathrm{~min}$, washing the precipitated cells for three times with distilled water, and drying at $60{ }^{\circ} \mathrm{C}$ until it reached to a constant weight [26]. 


\subsection{Measurement of GA-Me}

The GA-Me yield (mg. $\mathrm{L}^{-1}$ ) includes the contents of GA-Me from both mycylia and fermented broth. The determination of GA-Me was made by a similar method described in a previous work [22]. The dried mycelia ( $2 \mathrm{~g}$ ) were extracted by circumfluence with $70 \%(\mathrm{v} / \mathrm{v})$ ethanol $(100 \mathrm{~mL})$ for $2 \mathrm{~h}$ (twice), and ultrasonic treatment for $1 \mathrm{~h}$ (three times). The supernatant was dried at $50{ }^{\circ} \mathrm{C}$ under vacuum, the residues were suspended in water, and redissolved in absolute ethanol for high performance liquid chromatography (HPLC) analysis. For treatment of GA-Me samples from fermented broth, after removal of mycelia by centrifugation, the supernatant was dried at $50{ }^{\circ} \mathrm{C}$ under vacuum, and the residues were suspended in water, and redissolved in absolute ethanol for HPLC analysis. The analysis was perfomed on a Agilent 1200 series HPLC instrument $(5 \mu \mathrm{m}$ Agilent Zorbax SB-C18 column, $250 \times 4.6 \mathrm{~mm}$ ). The elution was performed at a flow rate of $1.0 \mathrm{~mL} \cdot \mathrm{min}^{-1}$ with a linear gradient of solvent A (methanol/acetic acid, 100:0.5, v/v) and solvent B (water). The gradient, starting at sample injection, was linear from $80 \%$ to $100 \%$ A in $20 \mathrm{~min}$ and the elution continued for an additional $20 \mathrm{~min}$ at $100 \% \mathrm{~A}$. The sample injection volume was $20 \mu \mathrm{L}$, GA-Me was detected at $245 \mathrm{~nm}$. Standard curves of peak area, as a function of the concentration of GA-Me was prepared for quantitative analysis [22]. The standard of GA-Me was extracted and purified from mycelia with preparative liquid chromatography in our lab with purity over $96 \%$.

\subsection{RSM Experimental Design and Statistical Analysis}

The preliminary trails indicated that glucose, peptone and fermentation time were the significant variables for GA production. Hence, these three variables were chosen to obtain the optimum levels. A central composite design (CCD) was used in the optimization of GA-Me production. Table 1 shows the ranges and the levels of the variables. The lowest and the highest levels of variables were: glucose, 36.59 and $53.41 \mathrm{~g} \cdot \mathrm{L}^{-1}$; peptone 3.32 and $6.68 \mathrm{~g} \cdot \mathrm{L}^{-1}$; time, 265.91 and $434.09 \mathrm{~h}$. All variables were taken at a central coded value considered as zero. The CCD contains a total of 20 experiments with the first 14 experiments organized in a fractional factorial design, with the experimental trails from 15 to 20 involving the replications of the central points (Table 2). A mathematical model, describing the relationships between the process indice (the yield of GA-Me) and the fermentation parameters in second-order equation, was developed. The yield of GA-Me by G. lucidum was multiply regressed with respect to the fermentation parameters by the least squares method (Equation (2)) as follows:

$$
Y_{i}=\beta_{0}+\sum \beta_{i} x_{i}+\sum \beta_{i i} x_{i}^{2}+\sum \beta_{i j} x_{i} x_{j}
$$

where $Y_{i}$ is the predicted response variable; $\beta_{0}, \beta_{i}, \beta_{i i}, \beta_{i j}$ are constant regression coefficients of the model, and $x_{i}, x_{j}(i=1,3 ; j=1,3, I \neq j)$ represent the independent variables (fermentation parameters) in the form of coded values.

The accuracy and general ability of the above polynomial model could be evaluated by the coefficient of determination $R^{2}$. The significance of each coefficient was determined using Student's t-test.

The SAS statistical package [27] was used for regression and graphical analysis of data obtained. The optimum levels of glucose, peptone and fermentation time were obtained by solving the regression equation. 


\section{Conclusions}

Enhanced production of individual GA-Me, an important bioactive triterpene, by G. lucidum in submerged culture was studied. From the present study, it is evident that the use of a statistical culture condition optimization approach and response surface methodology was helpful to locate the optimum levels of the significant conditions with minimum effort and time. The optimization of the culture conditions not only resulted in a 129.6\% higher GA-Me concentration than using non-optimized medium, but also in a successful large scale fermentation process. In addition, GA-Me is not only present in G. lucidum mycelia but also in fermented broth.

\section{Acknowledgments}

Thanks the financially support by the National "948" Project of China (Project No. 2012-4-11) and the National Natural Science Foundation of China (Project No. 30700552).

\section{References}

1. Liu, G.Q.; Zhang, K.C. Mechanisms of the anticancer action of Ganoderma lucidum (Leyss. ex. Fr.) Karst.: A new understanding. J. Integr. Plant Biol. 2005, 47, 129-135.

2. Cheung, W.M.W.; Hui, W.S.; Chu, P.W.K.; Chiu, S.W.; Ip, N.Y. Ganoderma extract activates MAP kinases and induces the neuronal differentiation of rat pheochromocytoma PC12 cells. FEBS Lett. 2000, 486, 291-296.

3. Gao, J.J.; Min, B.S.; Ahn, E.M.; Nakamura, N.; Lee, H.K.; Hattori, M. New triterpene aldehydes, lucialdehydes A-C, from Ganoderma lucidum and their cytotoxicity against murine and human tumor cells. Chem. Pharm. Bull. 2002, 50, 837-840.

4. Ha, T.B.T.; Gerhauser, C.; Zhang, W.D.; Ho-Chong-Line, N.; Fouraste, I. New lanostanoids from Ganoderma lucidum that induce NAD(P)H: Quinone oxidoreductase in cultured hepalclc7 murine hepatoma cells. Planta Med. 2000, 66, 681-684.

5. Gao, Y.H.; Zhou, S.F.; Jiang, W.Q.; Huang, M.; Dai, X.H. Effects of Ganopoly(R) (a Ganoderma lucidum polysaccharide extract) on the immune functions in advanced-stage cancer patients. Immunol. Invest. 2003, 32, 201-215.

6. Miyamoto, I.; Liu, J.; Shimizu, K.; Sato, M.; Kukita, A.; Kukita, T.; Kondo, R. Regulation of osteoclastogenesis by ganoderic acid DM isolated from Ganoderma lucidum. Eur. J. Pharmacol. 2009, 602, 1-7.

7. Chen, N.H.; Liu, J.W.; Zhong, J.J. Ganoderic acid T inhibits tumor invasion in vitro and in vivo through inhibition of MMP expression. Pharmacol. Rep. 2010, 62, 150-163.

8. Chen, Y.S.; Ming, S.S.; Cheng, T.W. Differential effects of ganodermic acid S on the thromboxane A2-signaling pathways in human platelets. Biochem. Pharmacol. 1999, 58, 587-595.

9. Kimura, Y.; Taniguchi, M.; Baba, K. Antitumor and antimetastatic effects on liver of triterpenoid fractions of Ganoderma lucidum: Mechanism of action and isolation of an active substance. Anticancer Res. 2002, 22, 3309-3318. 
10. Min, B.S.; Gao, J.J.; Nakamura, N.; Hattori, M. Triterpenes from the spores of Ganoderma lucidum and their cytotoxicity against meth-A and LLC tumor cells. Chem. Pharm. Bull. 2000, 48, 1026-1033.

11. Xu, J.W.; Zhao, W.; Zhong, J.J. Biotechnological production and application of ganoderic acids. Appl. Microbiol. Biotechnol. 2010, 87, 457-466.

12. Wang, G.; Zhao, J.; Liu, J.W.; Huang, Y.; Zhong, J.J.; Tang, W. Enhancement of IL-2 and IFN- $\gamma$ expression and NK cells activity involved in the anti-tumor effect of ganoderic acid Me in vivo. Int. Immunopharmacol. 2007, 7, 864-870.

13. Chen, N.H.; Liu, J.W.; Zhong, J.J. Ganoderic acid Me inhibits tumor invasion through down-regulating matrix metalloproteinases $2 / 9$ gene expression. J. Pharmacol. Sci. 2007, 108, 212-216.

14. Chen, N.H.; Zhong, J.J. Ganoderic acid Me induces G1 arrest in wild-type p53 human tumor cells while G1/S transition arrest in p53-null cells. Process Biochem. 2009, 44, 928-933.

15. Komoda, Y.; Shimizu, M.; Sonoda, Y.; Sato, Y. Ganoderic acid and derivatives as cholesterol synthesis inhibitors. Chem. Pharm. Bull. 1989, 37, 531-533.

16. Fang, Q.H.; Zhong, J.J. Two-stage culture process for improved production of ganoderic acid by liquid fermentation of higher fungus Ganoderman lucidum. Biotechnol. Progr. 2002, 18, 51-54.

17. Liu, G.Q.; Xiao, H.X.; Wang, X.L.; Zhao, Y.; Zhang, Y.G.; Ren, G.P. Stimulated production of triterpenoids of Ganoderma lucidum by an ether extract from the medicinal insect, Catharsius molossus and identification of the key stimulating active components. Appl. Biochem. Biotechnol. 2011, 165, 87-97.

18. Tang, Y.J.; Zhang, W.; Zhong, J.J. Performance analyses of a pH-shift and DOT-shift integrated fed-batch fermentation process for the production of ganoderic acid and Ganoderma polysaccharides by medicinal mushroom Ganoderma lucidum. Bioresource Technol. 2009, 100, 1852-1859.

19. Zhang, W.X.; Zhong, J.J. Effect of oxygen concentration in gas phase on sporulation and individual ganoderic acids accumulation in liquid static culture of Ganoderma lucidum. J. Biosci. Bioeng. 2010, 109, 37-40.

20. Wagner, R.; Mitchell, D.A.; Sassaki, G.L.; De Almeida Amazonas, M.A.L.; Berovic, M. Current techniques for the cultivation of Ganoderma lucidum for the production of biomass, ganoderic acid and polysaccharides. Food Technol. Biotechnol. 2003, 41, 371-382.

21. Xu, P.; Ding, Z.Y.; Qian, Z.; Zhao, C.X.; Zhang, K.C. Improved production of mycelial biomass and ganoderic acid by submerged culture of Ganoderma lucidum SB97 using complex media. Enzyme Microb. Technol. 2008, 42, 325-331.

22. Xu, J.W.; Xu, Y.N.; Zhong, J.J. Production of individual ganoderic acids and expression of biosynthetic genes in liquid static and shaking cultures of Ganoderma lucidum. Appl. Microbiol. Biotechnol. 2010, 85, 941-948.

23. Liu, G.Q.; Ding, C.Y.; Zhang, K.C. Effects of powdered dung beetle (Catharsius molossus) on cell growth and triterpenoid production of Ganoderma lucidum. Mycosystema 2008, 27, 757-762.

24. Baskar, G.; Rajesh, L.K.S.; Pavithra, S.K.; Aarathi, S.; Renganathan, S. Production of ganoderic acid by Ganoderma lucidium MTCC 1039 from cottonseed oil cake, Statistical screening of process variables. Indian J. Biotechnol. 2011, 10, 65-69. 
25. Gong, H.G.; Zhong, J.J. Hydrodynamic shear stress affects cell growth and metabolite production by medicinal mushroom Ganoderma lucidum. Chin. J. Chem. Eng. 2005, 13, 426-428.

26. Liu, G.Q.; Wang, X.L. Optimization of critical medium components using response surface methodology for biomass and extracellular polysaccharide production by Agaricus blazei. Appl. Microbiol. Biotechnol. 2007, 74, 78-83.

27. The SAS Statistical Package, version 8.1; an integrated system of software; SAS Institute Inc.: Cary, NC, USA, 2000.

Sample Availability: Not available.

(C) 2012 by the authors; licensee MDPI, Basel, Switzerland. This article is an open access article distributed under the terms and conditions of the Creative Commons Attribution license (http://creativecommons. org/licenses /by/3.0/). 